\title{
Structural parameters from ground-based observations of newly discovered globular clusters in NGC 5128
}

\author{
M. Gómez ${ }^{1}$, D. Geisler ${ }^{1}$, W. E. Harris ${ }^{2}$, T. Richtler ${ }^{1}$, G. L. H. Harris ${ }^{3}$, and K. A. Woodley ${ }^{2}$ \\ 1 Grupo de Astronomía, Depto. de Física, Universidad de Concepción, Casilla 160, Concepción, Chile \\ e-mail: matias@astro-udec.cl \\ 2 Department of Physics and Astronomy, McMaster University, Hamilton ON L8S 4M1, Canada \\ 3 Department of Physics, University of Waterloo, Waterloo, Ontario, N2L 3G1, Canada
}

Received 10 May 2005 / Accepted 17 October 2005

\section{ABSTRACT}

We have investigated a number of globular cluster candidates from a recent wide-field study by Harris et al. (2004a, AJ, 128, 712) of the giant elliptical galaxy NGC 5128. We used the Magellan I telescope + MagIC camera under excellent seeing conditions $\left(0.3^{\prime \prime}-0.6^{\prime \prime}\right)$ and obtained very high resolution images for a sample of 44 candidates. Of these, 15 appear to be bonafide globular clusters in NGC 5128 while the rest are either foreground stars or background galaxies. We also serendipitously discovered 18 new cluster candidates in the same fields. Our images allow us to study the light profiles of the likely clusters, all of which are well resolved. This is the first ground-based study of structural parameters for globular clusters outside the Local Group. We compare the psf-deconvolved profiles with King models and derive structural parameters, ellipticities and surface brightnesses. We compare the derived structural properties with those of other well-studied globular cluster systems. In general, our clusters are similar in size, ellipticity, core radius and central surface brightness to their counterparts in other galaxies, in particular those in NGC 5128 observed with HST by Harris et al. (2002, AJ, 124, 1435). However, our clusters extend to higher ellipticities and larger half-light radii than their Galactic counterparts, as do the Harris et al. sample. Combining our results with those of Harris et al. fills in the gaps previously existing in $r_{\mathrm{h}}-M_{V}$ parameter space and indicates that any substantial difference between presumed distinct cluster types in this diagram, including for example the Faint Fuzzies of Larsen \& Brodie (2000, AJ, 120, 2938) and the "extended, luminous" M 31 clusters of Huxor et al. $(2005$, MNRAS, 360, 1007) is now removed and that clusters form a continuum in this diagram. Indeed, this continuum now extends to the realm of the Ultra Compact Dwarfs. The metal-rich clusters in our sample have half-light radii that are almost twice as large in the mean as their metal-poor counterparts, at odds with the generally accepted trend. The possibility exists that this result could be due in part to contamination by background galaxies. We have carried out additional analysis to quantify this contamination. This shows that, although galaxies cannot be easily told apart from clusters in some of the structural diagrams, the combination of excellent image quality and Washington photometry should limit the contamination to roughly $10 \%$ of the population of cluster candidates. Finally, our discovery of a substantial number of new cluster candidates in the relatively distant regions of the NGC 5128 halo suggests that current values of the total number of globular clusters may be underestimates.

Key words. galaxies: individual: NGC 5128 - galaxies: star clusters - globular clusters: general

\section{Introduction}

At a distance of $\sim 4$ Mpc (Soria et al. 1996; Rejkuba 2004; Harris et al. 2004a), NGC 5128 (Centaurus A) is the closest giant elliptical ( $\mathrm{gE}$ ) galaxy (see Israel 1998 for a review). It possesses a rather low specific frequency of globular clusters (GCs), with $S_{N}=1.4 \pm 0.2$ (Harris et al. 2004b, hereafter $\mathrm{HHG}$ ), yet still hosts around $1000 \mathrm{GCs}$. This makes its globular cluster system (GCS) the largest of any galaxy known within $\sim 15 \mathrm{Mpc}$. It is therefore a prime target for studies of extragalactic GCSs. This is especially important given that systematic differences are suspected among GCSs depending on their host galaxy type and environment (Fleming et al. 1995; Kissler-Patig 2000). NGC 5128 offers a unique opportunity to study in great detail the GCS of a $\mathrm{gE}$ and to both compare it with closer systems, hosted only by late-type and dwarf galaxies, and to use it as a prototype for the GCSs of more distant gEs.

However, the study of the NGC 5128 GCS has been hampered by a set of observational circumstances which make further study far from straightforward. The low galactic latitude $\left(b=+19^{\circ}\right)$ makes the contamination by foreground stars a major issue. These, together with background galaxies, vastly outnumber the cluster population and many of them occupy a similar range in colour and magnitude, even if using a colour like the Washington $C-T_{1}$ index, which has proven especially powerful in distinguishing clusters from contaminating objects (Geisler et al. 1996; Dirsch et al. 2003). In their widefield Washington photometric investigation of NGC 5128, 
Harris et al. (2004a) estimated that bonafide GCs constitute only $\sim 1 \%$ of the $10^{5}$ objects they observed. In addition, NGC 5128 is so close that its GCS is very spread out in angular size, and some clusters have been found at distances as large as $40^{\prime}$ from the optical center (Harris et al. 1992; Peng et al. 2004), requiring the use of very wide field of view detectors for a comprehensive study. Yet it is distant enough that GCs cannot be easily told apart from the background and foreground population via image resolution, at least with typical groundbased images. With characteristic half-light radii of $0.3^{\prime \prime}-1.0^{\prime \prime}$ (Harris et al. 2002), excellent seeing conditions are needed to resolve the majority of the clusters from the ground.

The study of the structure of globular clusters has led to the discovery that they define a "fundamental plane" in analogy to that of elliptical galaxies. This is, they occupy a narrow region in multi-parameter space. This has been shown for Milky Way clusters (Djorgovski 1995) as well as for a few other GCSs in the Local Group (Djorgovski et al. 1997; Barmby et al. 2002; Larsen et al. 2002) and a sample of NGC 5128 GCs studied with HST by Harris et al. (2002). As the structure of a cluster is the result of its dynamical history, it is of great importance to compare cluster structures from a variety of galaxies along the Hubble sequence and to look for correlations with galaxy type. This is especially true in the case of elliptical galaxies, which have presumably a more complex formation history and are likely to have experienced several distinct formation events, as suggested by the usual bimodality in the colours of their GCSs (Geisler et al. 1996; Kundu \& Whitmore 2001; Larsen et al. 2001). The half-light radius $r_{\mathrm{h}}$ should remain roughly constant throughout the life of a GC (e.g. Spitzer \& Thuan 1972; Aarseth \& Heggie 1998), so its current size should reflect conditions of the proto-GC cloud. Any systematic variation of $r_{\mathrm{h}}$ within or among galaxies can provide insights into GC formation. For example, Mackey \& Gilmore (2004) found very different $r_{\mathrm{h}}$ distributions for disk/bulge, old halo and young halo Galactic GCs. Therefore, studying structural parameters of more GCSs and especially those of gE galaxies may help our understanding of galaxy formation.

In addition, a number of cluster subtypes have been suggested recently on the basis of their distinct properties. For example, Larsen \& Brodie (2000) find a class of extended, intermediate luminosity clusters in NGC 1023 which they refer to as "Faint Fuzzies". They also find similar objects in NGC 3384 (Larsen et al. 2001). Since these objects have so far only been identified in these two lenticular galaxies, it has recently been suggested (Burkert et al. 2005) that these objects form only in such galaxies and indeed that their formation may be intimately related to that of their parent galaxy. Similarly, Huxor et al. (2005) have discovered 3 very large, luminous GCs in the halo of M 31 which appear to be unique. In addition, a new type of Ultra Compact Dwarf (UCD) galaxy now appears to exist (Hilker et al. 1999; Drinkwater et al. 2002) which may or may not be related to GCs (Mieske et al. 2002). Haşegan et al. (2005) report the discovery of several bright objects in the Virgo Cluster which they refer to as DGTOs (Dwarf-Globular Transition Objects) and propose the use of the $\mathrm{M} / \mathrm{L}$ ratio to distinguish between bright GCs and UCDs. How unique are such objects? Do they exist in other galaxies, of other types? Are their properties truly distinct from those of other GCs or do GCs populate a continuum with no clear subclasses? What is the relation of GCs to the UCDs, if any? Such questions can be addressed by obtaining high quality structural parameters for as many different GCs in as many different types of galaxies as possible.

There have been intriguing hints (Kundu \& Whitmore 2001; Larsen et al. 2001; Larsen \& Brodie 2003) that the blue clusters in gEs are systematically larger by some $20 \%$ on average than their red counterparts, based on WFPC2 data. Recent Virgo Cluster ACS data (Jordán et al. 2005) strengthen this result. This seems to be a widespread effect, although its cause is still not clear. For example, it has been suggested that this effect is consistent with GCs having half mass radii distributions that do not depend on metallicity (Jordan 2004) or may simply reflect the fact that the red clusters are generally more centrally concentrated than their blue companions (e.g. Geisler et al. 1996) and that the larger tidal forces there lead to more compact clusters in the inner regions (Larsen \& Brodie 2003).

Using WFPC2 data, Harris et al. (2002) obtained images for $27 \mathrm{GCs}$ and derived their structural parameters. Combining with similar data for inner halo clusters from Holland et al. (1999), they found that the light profiles fit classic King models very well and that their structural parameters were similar to those of MW GCs, although their ellipticities were substantially larger than those of MW GCs and much more like those of M 31 clusters.

Recently, Martini \& Ho (2004) have obtained the velocity dispersions for the brightest 14 clusters in NGC 5128. Combining these data with the Harris et al. (2002) structural parameters, they were able to construct the fundamental plane for the clusters and showed that they follow approximately the same relationships found for Local Group clusters. This, in spite of their extreme masses and luminosities (about 10 times larger than nearby counterparts). However, since the discovery of the first GC in NGC 5128 (Graham \& Phillips 1980), its known GC population has steadily increased and is estimated today to be $\sim 1000$ (Harris et al. 2004b), so one of course would like to extend such analysis towards less luminous clusters and study a much more representative sample before definitive conclusions can be reached.

In this paper, we report the observation of a number of small fields around NGC 5128 under exceptional seeing obtained at the Magellan I telescope. These images allowed us to resolve known cluster candidates (and thus confirm or discard their cluster nature on the basis of their resolution and shape) and to detect a number of new ones. We have used these high-resolution images to derive structural parameters, surface brightnesses and central mass densities, which we compare to those of other well-studied GCSs. These are the first structural parameters derived for GCs beyond the Local Group using ground-based images.

The paper is organised as follows. In Sect. 2, we present the observations and reductions and describe our procedure for identifying clusters. In Sect. 3, we derive the structural parameters by fitting to models. In Sect. 4, we discuss our results and compare the derived structural parameters with those of other 


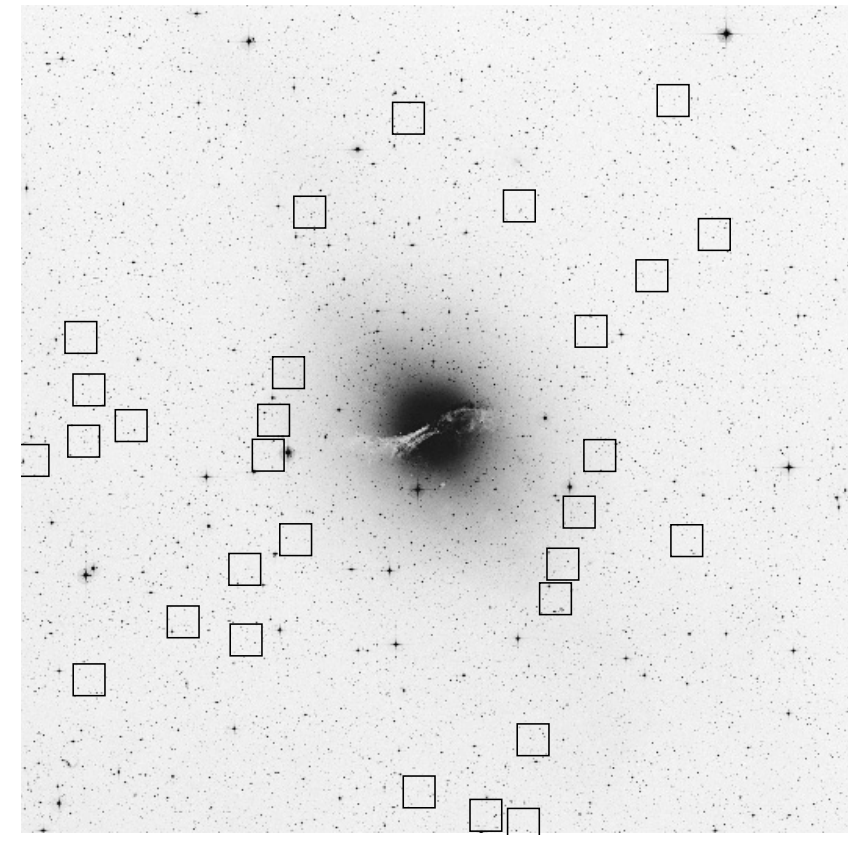

Fig. 1. A one square-degree image from DSS. Overlaid are 29 of the 30 fields observed with MagIC (one is slightly off this FOV) in actual size $\left(2.3^{\prime} \times 2.3^{\prime}\right)$. The orientation is $\mathrm{N}$ up, E to the left.

GCSs. We discuss the contamination by background galaxies and its effects in Sect. 5. Finally, we summarize our major findings in Sect. 6.

\section{The data}

\subsection{Observations}

The fields were selected from the sample of Harris et al. (2004a). They present a list of 327 candidate GCs based on colour and incipient resolution on their CTIO 4m BTC (Big Throughput Camera) frames with seeing $\sim 1.2^{\prime \prime}$ and pixel scale of $0.42^{\prime \prime} /$ pix. On the basis of brightness, we selected 30 fields, which contain about 45 of these candidates within the field of view of MagIC (Magellan Instant Camera). We concentrated on clusters in the distant halo of the galaxy in order to explore their nature in greater detail. These candidates had not been observed in previous spectroscopic work, thus their true nature was unknown.

Images were obtained with the Magellan I $6.5 \mathrm{~m}$ telescope at Las Campanas Observatory on Jan. 30, 31 and Feb. 1, 2004 with MagIC. This is a $2 \mathrm{~K} \times 2 \mathrm{~K} \mathrm{CCD}$ with a scale of $0.069^{\prime \prime} /$ pix (very similar to that of the PC on WFPC2), spanning a field of view of $2.3^{\prime} \times 2.3^{\prime}$. All of the fields (overlaid in Fig. 1) were observed at least once in $R$ and a few of them also in $B$. For these latter observations (which were taken during the third night) the seeing was significantly worse (over $0.7^{\prime \prime}$ ). Therefore, our results refer only to the $R$ frames, in which we enjoyed superb seeing of $0.3-0.6^{\prime \prime}$. Typical exposure times were 180-300 s. Tables 1 and 2 give details of the observations and observed clusters. The nights were not photometric but none of our results depends on absolute photometry acquired during this run.

\subsection{Data reduction}

The frames were bias-subtracted and flatfield corrected using the IRAF $^{1}$ script MagIC-tools, as described by Phillips (2002). For a few cases, the fields were observed twice. Those frames with comparable seeing were combined using imcombine after the alignment with geomap and geotrans with respect to the frame with higher $\mathrm{S} / \mathrm{N}$. No cosmic rays were detected near or on the cluster candidates, hence no attempt was made to remove them. Bad pixels were replaced by interpolation with neighbouring pixels using the task fixpix.

\subsection{Cluster identification}

The globular clusters in NGC 5128 have characteristic halflight radii of $0.3^{\prime \prime}-1^{\prime \prime}$ (measured from HST imaging, Harris et al. 2002), and so with sub-arcsecond seeing quality the great majority of the clusters should be distinguished from stars and large and/or irregular and/or elliptical galaxies. Given our excellent seeing and the high resolution of MagIC, we were not only able to achieve this but also discovered many new faint candidates that serendipitously lie in the same fields. This was done by subtracting the stellar PSF from all sources in each frame and visually inspecting the residuals. DAOPHOT II (Stetson 1987) was run under IRAF first to detect the sources with the daofind algorithm. Aperture photometry was then performed with a radius of 4 pixels. This was the basis to build the PSF by using $\sim 30$ bright, isolated stars per frame. Cluster candidates had broader profiles and we are confident that they were not used for the PSF. Anyway, the large number of stars used for the PSF would blur any effect of including possible compact clusters into this category.

The non stellar appearance of the cluster candidates allowed a straightforward identification. After subtraction of the PSF, resolved objects (both NGC 5128 GCs as well as certain types of background galaxies) leave a "doughnut-shape", being undersubtracted in the wings and oversubtracted in the center, as shown in Fig. 2. Resolved objects were further culled by eliminating those that had very large and/or irregular profiles in order to weed out as many galaxies as possible. However, relatively small, regular, round galaxies will not be recognized and may contaminate our sample.

After a visual inspection of the resulting images, we found that, starting from 44 cluster candidates, only 17 of them (39\%) had cluster-like residuals. Twelve of the 44 were not resolved and show a star-like profile, and 15 are background galaxies. This illustrates the general severe problem of field contamination discussed above: even after careful selection of candidates from their colors and (in some cases) barely extended appearance on good-quality ground-based images, the total number of non-cluster objects in the list is larger than the cluster population we are trying to find.

In addition, we could identify several new fainter candidates in our MagIC fields, typically 2-3 per frame. For the

\footnotetext{
1 IRAF is distributed by the National Optical Astronomy Observatories, which are operated by the Association of Universities for Research in Astronomy, Inc., under cooperative agreement with the National Science Foundation.
} 
Table 1. Washington photometry and classification of the 44 observed targets (from Harris et al. 2004a). The first column gives their identification. Objects in common with Woodley et al. (2005) are indicated by WHH and their classification (star/galaxy) or cluster number. The coordinates $(\alpha, \delta)$ are given in the second and third column. The colours in the Washington system from Harris et al. (2004a) are listed in the following three columns. The last column indicates our classification, according to their morphology in our MagIC frames.

\begin{tabular}{|c|c|c|c|c|c|c|}
\hline $\begin{array}{l}\text { Object ID } \\
(\mathrm{HHG})\end{array}$ & $\alpha_{2000}$ & $\delta_{2000}$ & $T_{1}$ & $M-T_{1}$ & $C-T_{1}$ & Classification \\
\hline 017 (WHH star) & 201.586426 & -43.15366 & 17.447 & 0.644 & 1.176 & star \\
\hline 021 & 201.627686 & -43.01647 & 17.546 & 0.568 & 1.433 & star \\
\hline 022 (WHH001) & 201.089172 & -43.04356 & 17.630 & 0.704 & 1.484 & cluster + star \\
\hline 032 & 200.909683 & -42.77305 & 17.984 & 0.740 & 1.462 & cluster \\
\hline 034 & 201.629135 & -43.01801 & 18.001 & 0.636 & 1.524 & star \\
\hline 036 (WHH star) & 201.010620 & -42.82483 & 18.046 & 0.724 & 1.586 & star \\
\hline 037 & 200.983215 & -42.61264 & 18.083 & 1.134 & 2.588 & star \\
\hline 038 (WHH star) & 201.637817 & -43.05389 & 18.092 & 0.526 & 1.088 & star \\
\hline 039 & 201.670975 & -43.27308 & 18.108 & 0.803 & 1.563 & star \\
\hline 049 & 201.773285 & -43.25498 & 18.365 & 0.923 & 2.461 & galaxy \\
\hline 050 & 200.926392 & -43.16050 & 18.490 & 0.791 & 1.630 & star \\
\hline 051 (WHH004) & 201.169159 & -43.22168 & 18.531 & 0.868 & 1.927 & cluster \\
\hline 060 & 201.638031 & -43.04905 & 18.667 & 1.078 & 2.082 & star \\
\hline 068 & 201.231567 & -42.74451 & 18.765 & 0.743 & 1.770 & galaxy \\
\hline 069 & 201.639236 & -43.01849 & 18.770 & 0.541 & 1.198 & star \\
\hline 074 & 202.025970 & -43.06270 & 18.894 & 1.074 & 2.653 & galaxy \\
\hline 080 & 201.435043 & -42.64040 & 18.972 & 1.048 & 2.754 & cluster \\
\hline 081 & 201.134018 & -43.18247 & 18.992 & 0.764 & 1.470 & galaxy \\
\hline 084 & 200.956879 & -43.12678 & 19.023 & 0.885 & 2.166 & galaxy \\
\hline 086 (WHH031) & 201.672623 & -43.19029 & 19.048 & 0.846 & 1.618 & cluster \\
\hline 093 & 201.606171 & -42.95170 & 19.173 & 0.801 & 1.792 & cluster \\
\hline 094 (WHH gal.) & 201.117294 & -42.88461 & 19.191 & 0.978 & 1.965 & cluster + galaxy \\
\hline 098 & 201.188309 & -43.38884 & 19.255 & 0.824 & 1.660 & galaxy \\
\hline 099 & 201.099915 & -42.90296 & 19.266 & 0.679 & 1.270 & galaxy \\
\hline 102 & 201.263916 & -43.48138 & 19.351 & 0.916 & 2.200 & cluster \\
\hline 104 & 201.942627 & -43.03859 & 19.374 & 0.921 & 1.943 & cluster \\
\hline 105 & 201.576157 & -42.75731 & 19.377 & 0.879 & 1.865 & galaxy \\
\hline 106 (WHH029) & 201.591995 & -43.15297 & 19.379 & 0.788 & 1.538 & cluster \\
\hline 120 & 201.935272 & -42.97654 & 19.521 & 0.806 & 1.799 & galaxy \\
\hline 127 (WHH gal.) & 201.144211 & -43.21404 & 19.588 & 0.949 & 1.932 & cluster \\
\hline 128 & 201.100601 & -42.90571 & 19.598 & 0.903 & 1.939 & cluster \\
\hline 129 & 201.949738 & -42.91334 & 19.600 & 0.972 & 2.599 & cluster \\
\hline 130 & 202.090408 & -42.90478 & 19.602 & 0.753 & 1.649 & galaxy \\
\hline 141 & 201.157684 & -43.16919 & 19.720 & 1.000 & 2.053 & cluster \\
\hline 145 & 201.120895 & -43.11254 & 19.776 & 0.835 & 1.377 & galaxy \\
\hline 147 & 201.864517 & -43.01892 & 19.794 & 0.670 & 1.197 & galaxy \\
\hline 200 & 201.201157 & -43.47463 & 20.398 & 0.656 & 1.460 & star \\
\hline 208 & 201.398788 & -42.64296 & 20.467 & 1.176 & 2.235 & star \\
\hline 210 & 201.201355 & -43.47885 & 20.485 & 0.974 & 2.225 & cluster \\
\hline 225 & 201.375885 & -43.45494 & 20.725 & 1.001 & 1.745 & cluster \\
\hline 228 & 201.665817 & -43.27797 & 20.740 & 1.009 & 1.819 & galaxy \\
\hline 244 & 201.200668 & -43.50775 & 21.004 & 0.799 & 1.390 & galaxy \\
\hline 246 & 201.927948 & -43.32676 & 21.017 & 1.028 & & galaxy \\
\hline 327 & 201.620071 & -43.00220 & & & & cluster + star \\
\hline
\end{tabular}

majority of them, Washington photometry already exists from Harris et al. (2004a) and was used to apply the same color selection as they used to generate their candidate list. In all, 48 new candidates were found. Of these, 15 have very red colours and are presumably background galaxies and 11 had no photometry available. This happened either because they were too faint or they were located in the gaps between the BTC frames, where the photometry data come from. Thus, 22 new, good cluster candidates have been added to the analysis. The comparison with previous work shows that three of them were 
Table 2. Washington photometry and observational details for all of our final GC candidates. Clusters with no prefix are candidates from Harris et al. (2004a) database (from Table 1). Those labelled as "N" are newly identified objects in this study (see text for detail) WHH objects are those also present in Woodley et al. (2005). Note that clusters 022 and N3 were observed twice.

\begin{tabular}{|c|c|c|c|c|c|c|c|c|}
\hline $\begin{array}{l}\text { Cluster ID } \\
(\mathrm{HHG})\end{array}$ & $\alpha_{2000}$ & $\delta_{2000}$ & $\bar{T}$ & $\overline{C-T_{1}}$ & date & Airmass & $\begin{array}{l}\text { Exp. time } \\
\text { (s) }\end{array}$ & $\begin{array}{c}\text { Seeing } \\
\left({ }^{\prime \prime}\right)\end{array}$ \\
\hline 022 (WHH001) & 201.089172 & -43.043560 & 17.630 & 1.484 & 30 Jan. & 1.08 & 180 & 0.32 \\
\hline 022 (WHH001) & 201.089172 & -43.043560 & 17.630 & 1.484 & 30 Jan. & 1.07 & 300 & 0.35 \\
\hline 032 & 200.909683 & -42.773048 & 17.984 & 1.462 & 30 Jan. & 1.14 & 300 & 0.38 \\
\hline 051 (WHH004) & 201.169159 & -43.221680 & 18.531 & 1.927 & 30 Jan. & 1.05 & 180 & 0.38 \\
\hline 080 & 201.435043 & -42.640400 & 18.972 & 2.754 & 31 Jan. & 1.14 & 180 & 0.53 \\
\hline 086 (WHH031) & 201.672623 & -43.190289 & 19.048 & 1.618 & 31 Jan. & 1.09 & 180 & 0.59 \\
\hline 093 & 201.606171 & -42.951698 & 19.173 & 1.792 & 31 Jan. & 1.11 & 180 & 0.54 \\
\hline 102 & 201.263916 & -43.481380 & 19.351 & 2.200 & 30 Jan. & 1.04 & 300 & 0.35 \\
\hline 104 & 201.942627 & -43.038589 & 19.374 & 1.943 & 31 Jan. & 1.06 & 180 & 0.44 \\
\hline 106 (WHH029) & 201.591995 & -43.152969 & 19.379 & 1.538 & 31 Jan. & 1.12 & 180 & 0.50 \\
\hline 128 & 201.100601 & -42.905708 & 19.598 & 1.939 & $30 \mathrm{Jan}$. & 1.07 & 180 & 0.30 \\
\hline 129 & 201.949738 & -42.913342 & 19.600 & 2.599 & 31 Jan. & 1.06 & 240 & 0.43 \\
\hline 141 & 201.157684 & -43.169189 & 19.720 & 2.053 & 30 Jan. & 1.05 & 180 & 0.41 \\
\hline 210 & 201.201355 & -43.478851 & 20.485 & 2.225 & 30 Jan. & 1.04 & 180 & 0.39 \\
\hline 225 & 201.375885 & -43.454941 & 20.725 & 1.745 & 31 Jan. & 1.15 & 180 & 0.57 \\
\hline 327 & 201.620071 & -43.002201 & & & 31 Jan. & 1.11 & 180 & 0.53 \\
\hline N1 (C40) & 200.926392 & -43.160500 & 18.490 & 1.630 & 30 Jan. & 1.12 & 180 & 0.34 \\
\hline N3 & 201.072800 & -43.039169 & 20.897 & 1.848 & 30 Jan. & 1.08 & 180 & 0.32 \\
\hline N3 & 201.072800 & -43.039169 & 20.897 & 1.848 & 30 Jan. & 1.07 & 300 & 0.35 \\
\hline N5 & 201.136230 & -43.094910 & 20.774 & 1.680 & 30 Jan. & 1.06 & 300 & 0.30 \\
\hline N7 (PFF06) & 201.098740 & -43.131149 & 18.731 & 1.373 & 30 Jan. & 1.06 & 300 & 0.30 \\
\hline N8 & 201.103271 & -43.119518 & 20.057 & 1.654 & 30 Jan. & 1.06 & 300 & 0.30 \\
\hline N9 & 201.116486 & -43.109959 & 21.933 & 1.871 & 30 Jan. & 1.06 & 300 & 0.30 \\
\hline N10 (PFF09) & 201.130554 & -43.190781 & 19.258 & 1.474 & 30 Jan. & 1.05 & 180 & 0.41 \\
\hline N11 & 201.172958 & -43.214771 & 20.296 & 1.464 & 30 Jan. & 1.05 & 180 & 0.38 \\
\hline N12 & 201.140121 & -43.200439 & 20.119 & 1.568 & 30 Jan. & 1.05 & 180 & 0.38 \\
\hline N21 & 201.437943 & -42.649410 & 19.845 & 2.331 & 31 Jan. & 1.14 & 180 & 0.53 \\
\hline $\mathrm{N} 23$ & 201.561600 & -42.757729 & 20.072 & 0.815 & 31 Jan. & 1.13 & 240 & 0.49 \\
\hline N24 & 201.598083 & -43.151272 & 21.290 & 1.691 & 31 Jan. & 1.12 & 180 & 0.50 \\
\hline $\mathrm{N} 25$ & 201.571945 & -43.166100 & 20.914 & 2.207 & 31 Jan. & 1.12 & 180 & 0.50 \\
\hline N26 (PFF092) & 201.588715 & -42.955292 & 19.458 & 1.727 & 31 Jan. & 1.11 & 180 & 0.54 \\
\hline N30 & 201.675735 & -43.286640 & 19.916 & 1.247 & 31 Jan. & 1.09 & 180 & 0.57 \\
\hline N32 & 201.691513 & -43.194920 & 20.034 & 1.366 & 31 Jan. & 1.09 & 180 & 0.59 \\
\hline N33 & 201.686783 & -43.204910 & 20.156 & 1.887 & 31 Jan. & 1.09 & 180 & 0.59 \\
\hline N34 & 201.766739 & -43.259491 & 20.267 & 1.626 & 31 Jan. & 1.08 & 180 & 0.60 \\
\hline N35 & 201.787048 & -43.271809 & 21.005 & 1.957 & 31 Jan. & 1.08 & 180 & 0.60 \\
\hline N37 & 201.854248 & -43.002270 & 21.318 & 1.993 & 31 Jan. & 1.08 & 240 & 0.52 \\
\hline N41 & 201.974548 & -42.922680 & 19.988 & 2.515 & 31 Jan. & 1.06 & 240 & 0.43 \\
\hline N42 & 201.955078 & -42.932850 & 18.436 & 2.591 & 31 Jan. & 1.06 & 240 & 0.43 \\
\hline
\end{tabular}

already in the confirmed GC catalog of Peng et al. (2004), and one is listed as a GC by Hesser et al. (1984). They are marked in Tables 2 and 3 as "PFF" and "C" objects respectively and hence the number of truly new clusters is 18 .

This work therefore gives a total of 39 high-quality candidate clusters, 18 new and 21 previously existing candidates. After our imaging observations were procured, Woodley et al. (2005) obtained spectra for an independent sample of nearly a hundred of the HHG GC candidates and were able to classify them as bonafide NGC 5128 GCs, foreground stars or background galaxies on the basis of their radial velocities.
Nine objects are in common between the two studies and are accordingly labelled in Table 1 . Four of the objects identified as GCs by us are also GCs from their radial velocities, while two objects we classified as GCs (\#127 and \#94) are actually distant galaxies according to radial velocity (and labelled "WHH gal." in Table 1). In addition, there is perfect agreement in the (independent) classification for three foreground stars (see Table 1) We were not able to perform this comparison for our newly discovered cluster candidates since no other data exists for them besides the Harris et al. (2004a) photometry other than the fact that four of them were independently classified as 


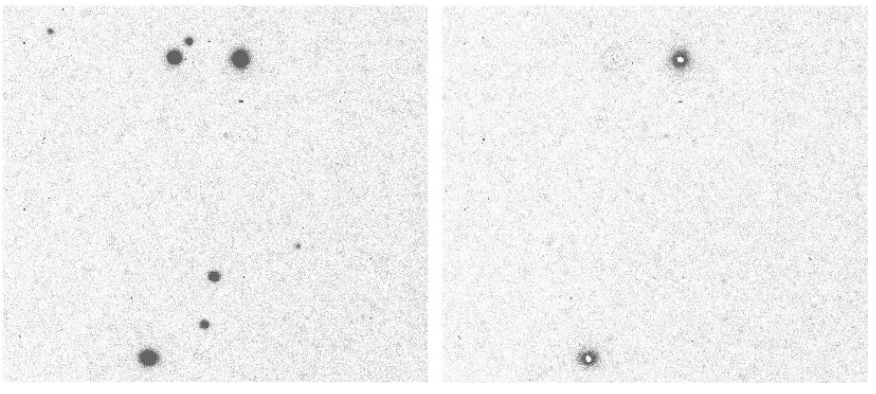

Fig. 2. Illustration of how the resolution technique works in discriminating resolved globular clusters in NGC 5128 from unresolved stars and also background galaxies. On the left is a small area $\left(37^{\prime \prime} \times 37^{\prime \prime}\right)$ of one of our MagIC images. On the right, one sees that after subtraction of a stellar psf, stars disappear while globular clusters (two of which are shown) appear as round doughnuts with oversubtracted centers and undersubtracted wings.

GCs based on lower resolution images. This test indicates that galaxies still contaminate our final GC sample, as expected. A much more detailed estimate of galaxy contamination is given in Sect. 5.

Although we have eliminated the two galaxies 94 and 127 from further consideration, both of them provide interesting properties. Object 127 does not deviate significantly from a typical King profile and all derived parameters (as well as photometry) are in the range of GCs. Perhaps its small core and effective radii could be the only indication of its strangeness. Object 94 was taken for a cluster in projection next to a distant galaxy but from its velocity, it might consist of a pair of galaxies, separated by $\sim 1.6^{\prime \prime}$. In Table 2 we present observational data for our new cluster candidates, labeled with an "N" prefix, and present the full list of our final cluster candidates.

\section{Analysis}

\subsection{Fit to the light profiles}

All of our final cluster candidates have FWHMs significantly larger than those of stars, thus the residuals are noticeable. No identification was attempted for faint objects, as they were beyond the limiting magnitude of the Harris et al. (2004a) database $(R \sim 22 \mathrm{mag})$.

For all objects listed in Table 2, a 2-D fitting was performed to derive their morphological parameters after deconvolution with the stellar PSF. This was done using the task ishape under BAOLAB ${ }^{2}$ (Larsen 1999). In two cases, a star was located $\sim 2-3^{\prime \prime}$ away from the cluster candidate. They were first removed using PSF-fitting and then ishape was run on the clean image.

Ishape convolves a model for the light distribution with the PSF and then performs a 2-D fitting with the actual profile by means of a weighted least-square minimization until the best fit

\footnotetext{
${ }^{2}$ BAOLAB is available at

http://www. astro.ku.dk/ soeren/baolab
}

parameters are obtained. For the latter we have chosen a King profile (King 1962), which has the form:

$$
\mu(r)=k\left[\frac{1}{\sqrt{1+r^{2} / r_{\mathrm{c}}^{2}}}-\frac{1}{\sqrt{1+r_{\mathrm{t}}^{2} / r_{\mathrm{c}}^{2}}}\right]^{2}, r<r_{\mathrm{t}} .
$$

Ishape also offers other profiles. Among them, the "Moffat-type" is the most frequently used (see Elson et al. 1987; Larsen 2001; Mackey \& Gilmore 2003). Although we extensively tried different models and parameters, the best results were obtained with King profiles, except for a very few cases which were better fit by a Moffat model and which are presumably background galaxies (see discussion).

King profiles are known to provide excellent fits to Milky Way GCs and are characterised by a tidal radius $r_{\mathrm{t}}$ and a core radius $r_{\mathrm{c}}$. Alternatively, a concentration parameter $c=r_{\mathrm{t}} / r_{\mathrm{c}}$ can be defined, which we use here. Note that this definition differs from the more familiar $c=\log \left(r_{\mathrm{t}} / r_{\mathrm{c}}\right)$. We prefer to use the first definition to keep consistency with the outputs from ishape. The $c$ parameter can be kept fixed or allowed to vary during the fitting process and is the most uncertain of the fitted parameters (Larsen 2001). However, a number of tests with our images show that, in fact, $c$ is stable typically within $20 \%$ when the radius of the fit increases from 15 to 25 pixels. This is encouraging given the large errors found in the literature. On the other hand, one should keep in mind the corresponding uncertainty in the tidal radii, which are derived directly from $c$ and $r_{\mathrm{c}}$.

The fit radius was 25 pixels or $1.725^{\prime \prime}$, corresponding to $33 \mathrm{pc}$ if a distance of $4 \mathrm{Mpc}$ is assumed $\left(1^{\prime \prime} \sim 19 \mathrm{pc}\right)$. Smaller radii were tried, but the subtracted images showed noticeable residuals in the wings. With the sole exception of $c$, the derived parameters do not change significantly with fitting radius. Figure 3 shows a typical example for one of the known cluster candidates (HHG128).

Formally, the lowest $\chi^{2}$ is achieved with $c$ as a free parameter. However, as this is the most uncertain of the parameters if left free, we have used fixed values of $c=30$ and $c=100$ to estimate the actual errors using these plausible values for clusters in our Galaxy (Djorgovski \& Meylan 1993). Hence, all derived sizes, ellipticities and position angles (PA) are the average from the fits assuming King models with $c=30,100$ and free. Their corresponding errors are the $\sigma$ in each case.

Ishape does not return either the core radius $r_{\mathrm{c}}$ or the tidal radius $r_{\mathrm{t}}$. Instead, some assumptions must be made to convert the given $F W H M$ and concentration parameter into these more familiar quantities. A discussion of this can be found in Larsen (2001) and Larsen (2004), where both a relation between the $F W H M$ and $r_{\mathrm{c}}$ and a numerical approximation for the effective (half-light) radius $r_{\mathrm{e}}$ are presented:

$$
\begin{aligned}
& F W H M=2\left[\left[\sqrt{1 / 2}+\frac{1-\sqrt{1 / 2}}{\sqrt{1+c^{2}}}\right]^{-2}-1\right]^{1 / 2} r_{\mathrm{c}} \\
& r_{\mathrm{e}} / r_{\mathrm{c}} \approx 0.547 c^{0.486} .
\end{aligned}
$$

The latter is good to $\pm 2 \%$ when $c>4$. These relations are valid when the King profiles are circularly symmetric. In our case 
Table 3. Results from the fitting procedure. The first column is the identification of the cluster. Then follow the core radius $r_{\mathrm{c}}$ in pc (assuming $d=4 \mathrm{Mpc}$ ), ellipticity and the position angle (PA). Column 5 gives the concentration parameter $c\left(=r_{\mathrm{t}} / r_{\mathrm{c}}\right)$. The effective or half-light radius $r_{\mathrm{e}}$ (in pc) is given in Col. 6. Column 7 lists the projected galactocentric distance in kpc, using $\alpha=201.364995$ and $\delta=-43.01917$ as the center of NGC 5128. The central surface brightness in $V, \mu_{0}(V)$ is listed in Col. 8 (see text).

\begin{tabular}{|c|c|c|c|c|c|c|c|}
\hline $\begin{array}{l}\text { Cluster ID } \\
(\mathrm{HHG})\end{array}$ & $\begin{array}{c}r_{\mathrm{c}} \\
(\mathrm{pc})\end{array}$ & $e$ & $\begin{array}{r}\text { PA } \\
(\mathrm{deg})\end{array}$ & $c$ & $\begin{array}{c}r_{\mathrm{e}} \\
(\mathrm{pc})\end{array}$ & $\begin{array}{l}R_{\mathrm{gc}} \\
(\mathrm{kpc})\end{array}$ & $\begin{array}{c}\mu_{0}(V) \\
\left(\operatorname{arcsec}^{-2}\right)\end{array}$ \\
\hline 022 (WHH001) & $0.73 \pm 0.39$ & $0.12 \pm 0.00$ & $2 . \pm 0.6$ & 61.9 & $2.96 \pm 1.58$ & 14.2 & 13.85 \\
\hline 022 (WHH001) & $0.75 \pm 0.39$ & $0.12 \pm 0.01$ & 1. \pm 1.4 & 58.0 & $2.94 \pm 1.53$ & 14.2 & 13.88 \\
\hline 032 & $1.25 \pm 0.44$ & $0.30 \pm 0.00$ & $-21 . \pm 0.1$ & 44.8 & $4.36 \pm 1.53$ & 29.0 & 15.23 \\
\hline 051 (WHH004) & $0.41 \pm 0.54$ & $0.00 \pm 0.01$ & 53. \pm 13.7 & 317.5 & $3.73 \pm 4.81$ & 17.4 & 14.01 \\
\hline 080 & $3.32 \pm 0.32$ & $0.08 \pm 0.01$ & $32 . \pm 4.6$ & 36.4 & $10.42 \pm 1.01$ & 26.7 & 18.38 \\
\hline 086 (WHH031) & $2.14 \pm 0.37$ & $0.24 \pm 0.00$ & $31 . \pm 0.5$ & 35.5 & $6.62 \pm 1.16$ & 19.7 & 17.34 \\
\hline 093 & $2.67 \pm 0.29$ & $0.18 \pm 0.00$ & $-48 . \pm 0.1$ & 43.1 & $9.08 \pm 1.00$ & 13.3 & 17.96 \\
\hline 102 & $1.99 \pm 2.95$ & $0.38 \pm 0.02$ & $11 . \pm 0.7$ & 30.0 & $5.69 \pm 8.41$ & 32.7 & 17.61 \\
\hline 104 & $3.18 \pm 0.25$ & $0.11 \pm 0.00$ & $-18 . \pm 1.0$ & 21.4 & $7.71 \pm 0.61$ & 29.6 & 18.39 \\
\hline 106 (WHH029) & $0.63 \pm 0.46$ & $0.00 \pm 0.01$ & $-28 . \pm 22.6$ & 62.9 & $2.58 \pm 1.86$ & 14.9 & 15.31 \\
\hline 128 & $0.93 \pm 0.31$ & $0.07 \pm 0.00$ & 42. \pm 2.8 & 30.9 & $2.69 \pm 0.89$ & 15.7 & 16.20 \\
\hline 129 & $3.21 \pm 0.20$ & $0.28 \pm 0.00$ & $-90 . \pm 0.9$ & 75.0 & $14.33 \pm 0.90$ & 30.8 & 18.99 \\
\hline 141 & $5.21 \pm 0.21$ & $0.06 \pm 0.01$ & $-65 . \pm 8.1$ & 46.5 & $18.42 \pm 0.77$ & 14.9 & 19.75 \\
\hline 210 & $3.97 \pm 0.37$ & $0.06 \pm 0.02$ & $-11 . \pm 23.8$ & 33.1 & $11.90 \pm 1.12$ & 33.2 & 20.06 \\
\hline 225 & $5.40 \pm 0.41$ & $0.19 \pm 0.02$ & $-17 . \pm 1.3$ & 43.9 & $18.55 \pm 1.42$ & 30.5 & 20.73 \\
\hline 327 & $0.71 \pm 0.51$ & $0.17 \pm 0.04$ & $-64 . \pm 2.4$ & 39.2 & $2.32 \pm 1.65$ & 13.1 & \\
\hline N1 (C40) & $1.38 \pm 0.38$ & $0.29 \pm 0.00$ & $79.8 \pm 0.4$ & 40.3 & $4.54 \pm 1.26$ & 24.5 & 15.94 \\
\hline N3 & $2.19 \pm 0.08$ & $0.02 \pm 0.01$ & $55.7 \pm 30.0$ & 34.6 & $6.69 \pm 0.25$ & 15.1 & 19.28 \\
\hline N3 & $2.09 \pm 0.16$ & $0.06 \pm 0.03$ & $-54.6 \pm 10.4$ & 50.8 & $7.71 \pm 0.59$ & 15.1 & 19.27 \\
\hline N5 & $1.62 \pm 0.21$ & $0.09 \pm 0.02$ & $-23.6 \pm 9.7$ & 22.4 & $4.00 \pm 0.53$ & 12.9 & 18.36 \\
\hline N7 (PFF06) & $1.06 \pm 0.44$ & $0.36 \pm 0.00$ & $88.8 \pm 0.8$ & 29.2 & $2.99 \pm 1.25$ & 15.7 & 15.45 \\
\hline $\mathrm{N} 8$ & $1.71 \pm 0.59$ & $0.38 \pm 0.01$ & $-81.7 \pm 0.0$ & 17.6 & $3.76 \pm 1.30$ & 15.1 & 17.64 \\
\hline N9 & $1.34 \pm 0.31$ & $0.00 \pm 0.04$ & $-90 . \pm 21.8$ & 24.6 & $3.47 \pm 0.80$ & 14.2 & 19.21 \\
\hline N10 (PFF09) & $1.13 \pm 0.37$ & $0.14 \pm 0.01$ & $85.3 \pm 0.9$ & 44.8 & $3.92 \pm 1.30$ & 17.0 & 16.29 \\
\hline N11 & $1.20 \pm 0.25$ & $0.13 \pm 0.01$ & $23.2 \pm 2.8$ & 34.1 & $3.64 \pm 0.78$ & 16.9 & 17.36 \\
\hline N12 & $1.25 \pm 0.28$ & $0.14 \pm 0.02$ & $-25.8 \pm 1.2$ & 22.9 & $3.14 \pm 0.70$ & 17.1 & 17.14 \\
\hline $\mathrm{N} 21$ & $3.32 \pm 0.32$ & $0.08 \pm 0.01$ & $31.7 \pm 4.6$ & 36.4 & $10.42 \pm 1.01$ & 26.1 & 19.15 \\
\hline $\mathrm{N} 23$ & $2.61 \pm 0.24$ & $0.23 \pm 0.00$ & $47.9 \pm 0.2$ & 43.2 & $8.89 \pm 0.82$ & 20.9 & 18.57 \\
\hline $\mathrm{N} 24$ & $2.40 \pm 0.17$ & $0.12 \pm 0.03$ & $-73 . \pm 20.2$ & 56.8 & $9.37 \pm 0.68$ & 15.1 & 19.90 \\
\hline $\mathrm{N} 25$ & $2.98 \pm 0.21$ & $0.08 \pm 0.02$ & 44. \pm 13.7 & 29.1 & $8.38 \pm 0.60$ & 14.8 & 19.94 \\
\hline N26 (PFF092) & $0.98 \pm 0.35$ & $0.07 \pm 0.01$ & $44.5 \pm 4.2$ & 55.8 & $3.79 \pm 1.34$ & 12.3 & 16.33 \\
\hline $\mathrm{N} 30$ & $2.27 \pm 0.26$ & $0.10 \pm 0.03$ & $11.2 \pm 19.9$ & 31.3 & $6.61 \pm 0.78$ & 24.5 & 18.20 \\
\hline N32 & $3.94 \pm 0.32$ & $0.12 \pm 0.01$ & $-8.1 \pm 3.1$ & 51.4 & $14.61 \pm 1.19$ & 20.7 & 19.43 \\
\hline N33 & $0.99 \pm 0.46$ & $0.01 \pm 0.03$ & $-3.2 \pm 26.4$ & 24.0 & $2.53 \pm 1.16$ & 21.0 & 16.76 \\
\hline N34 & $4.39 \pm 0.12$ & $0.09 \pm 0.00$ & $-23.4 \pm 9.1$ & 51.0 & $16.24 \pm 0.45$ & 26.5 & 19.91 \\
\hline N35 & $3.65 \pm 0.76$ & $0.17 \pm 0.03$ & $-77.3 \pm 4.2$ & 71.1 & $15.84 \pm 3.31$ & 27.8 & 20.45 \\
\hline N37 & $4.10 \pm 0.21$ & $0.09 \pm 0.04$ & $52.6 \pm 21.2$ & 39.3 & $13.35 \pm 0.70$ & 25.1 & 20.91 \\
\hline N41 & $2.41 \pm 0.16$ & $0.24 \pm 0.01$ & $-40.9 \pm 0.0$ & 49.7 & $8.80 \pm 0.60$ & 31.9 & 18.80 \\
\hline $\mathrm{N} 42$ & $2.64 \pm 0.20$ & $0.09 \pm 0.00$ & $-39.1 \pm 4.3$ & 31.0 & $7.65 \pm 0.58$ & 30.7 & 17.35 \\
\hline
\end{tabular}

(2-D fit) the average of the $F W H M$ along the minor and major axis was used to compute $r_{\mathrm{c}}$ and $r_{\mathrm{e}}$. The values are listed in Table 3.

The ellipticities (Col. 3 in Table 3 ) are measured at the $F W H M$ as $e=1-b / a$, where $a$ and $b$ are the semi-major and semi-minor axes respectively. Their values and comparison with other GCSs will be discussed in Sect. 4.1.

The central surface brightness of the clusters can be derived assuming that a King profile remains a good representation of the light distribution of the cluster towards the center, even if this part cannot be resolved by our images. The central surface brightness $\mu_{0}$ of a King profile and total luminosity $L(R)$ within a radius $R<r_{\mathrm{t}}$ are related by (Larsen 2001):

$\mu_{0}=k\left(1-\frac{1}{\sqrt{1+c^{2}}}\right)^{2}$

$L(R)=$

$\pi k\left(r_{\mathrm{c}}^{2} \ln \left(1+\frac{R^{2}}{r_{\mathrm{c}}^{2}}\right)+\frac{R^{2}}{1+c^{2}}-\frac{4 r_{\mathrm{c}}^{2}}{\sqrt{1+c^{2}}}\left(\sqrt{1+\frac{R^{2}}{r_{\mathrm{c}}^{2}}}-1\right)\right)$ 


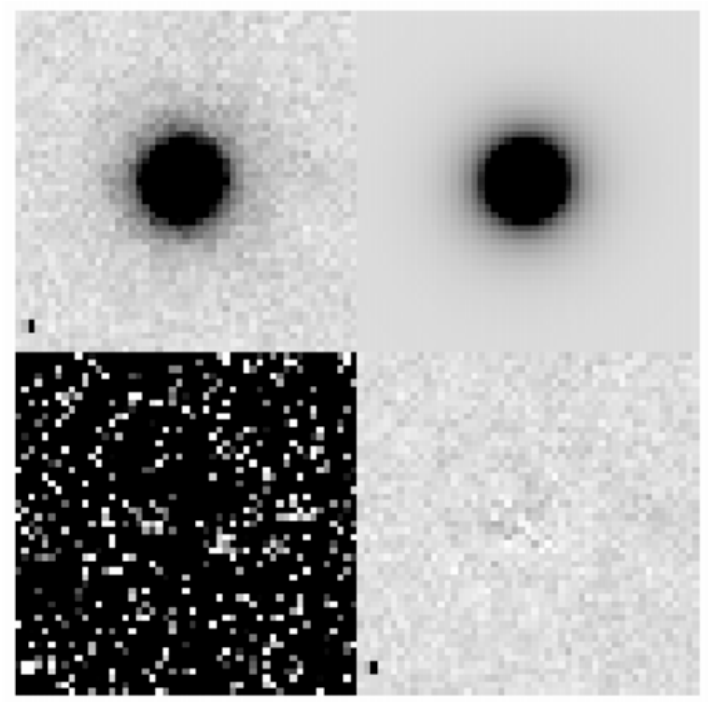

Fig. 3. Upper left: original image of the cluster candidate HHG128. Upper right: the model constructed using ishape and assuming a King profile, after the convolution with the PSF. Lower left: the weights assigned by ishape to each pixel. Lower right: the residuals after subtracting the original image with the model. Little structure is seen beyond the photon noise associated with the cluster's light.

Note that Eq. (4) is essentially a definition of the magnitude zeropoint parameter $k$; for the range of $c$-values of interest here, we have $k \simeq \mu_{0}$. $R$ was set to 20 pixels $\left(1.38^{\prime \prime}\right)$ and aperture photometry was consequently performed to compute the total luminosity $L(R)$ within this radius, which is roughly three times larger than the typical FWHM. As no Landolt standards were observed (since the nights were not photometric), we have used the Washington photometry from Harris (2004a), which includes virtually everything in the field of NGC 5128 in order to calibrate our photometry. For this purpose an aperture correction between their and our aperture radius had to be computed. The curves of growth (i.e. the $R$ magnitude vs. the aperture radius) show that this correction is $\Delta R=-0.130 \pm 0.005$. Our $R$ photometry can be directly compared to the $T_{1}$ filter as the difference is $\sim 0.01$ for old, globular cluster-like objects (see Geisler 1996). In addition to the aperture correction, we used $E_{B-V}=0.11$ (Schlegel et al. 1998) to correct for galactic absorption. Since all of our clusters are well away from the central dust lane, the reddening should be uniform.

The central surface brightness in the $R$ band for each cluster is thus derived using Eqs. (4) and (5). However, most values found in the literature are given only for the $V$ filter. Hence, we have taken an average colour of $V-R=0.5$ to estimate $\mu_{0}(V)$. The results are listed in Col. 8 of Table 3 . The spread in the intrinsic colour remains the main uncertainty for the values listed.

Two clusters (HHG022 and N3, see Tables 1-3) were observed twice under similar conditions. They allowed us to test the internal accuracy of the derived solutions. As can be seen from these tables, the results are encouraging given the very good agreement between the listed values. Furthermore, a much more telling - and fully independent - comparison is that

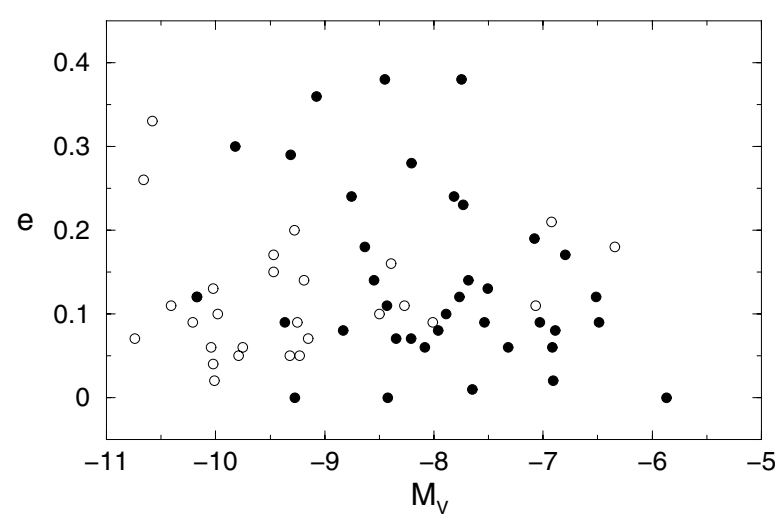

Fig. 4. The ellipticity $(e=1-b / a)$ as a function of the cluster's luminosity in the $V$ band, $M_{V}$. The same behaviour is observed for Galactic and M 31 clusters, in the sense that brighter clusters tend to be more flattened and there are no relatively faint, elliptical clusters. Our data are indicated by filled circles, while open circles represent the clusters from Harris et al. (2002).

with Harris et al. (2002). Their typical sizes and ellipticities are comparable to those derived by us.

\section{Discussion}

\subsection{Ellipticity}

The Galactic GCs show, on average, little elongation, apart from a few cases (e.g. $\omega$ Cen with $e=0.19$, Frenk \& Fall 1982). The range of ellipticities of GCs is interesting to study given that systematic differences have been found for other systems in the Local Group. Geisler \& Hodge (1980) concluded that only a few massive (especially intermediate age) clusters in the LMC are round, the large majority showing significant ellipticities. A similar feature was observed for GCs in M 31 (Barmby et al. 2002), where the brightest member G1 (=Mayall II) has $e=0.25 \pm 0.02$ measured from HST images (Rich et al. 1996). A common property of all these very elongated clusters is their high luminosity. In fact, the brightest GCs in our Galaxy, as well as in M31 and in the LMC, are the most flattened in their respective galaxies (van den Bergh 1984). Hesser et al. (1984) also noted that 4 of the 6 brightest known NGC 5128 GCs were noticeably flattened.

Although our sample is still small, we do observe a similar tendency of bright clusters to have higher ellipticities, as shown in Fig. 4. It is intriguing to suppose that such clusters may be the stripped nuclei of former nucleated dwarf elliptical galaxies (e.g. Martini \& Ho 2004), as is often supposed for $\omega$ Cen. However, note that the second most luminous Galactic GC (M54) is nearly spherical, although it is now generally regarded as the former nucleus of the Sgr dSph (e.g. Layden \& Sarajedini 2000).

The histogram of our ellipticities (Fig. 5) is in very good agreement with the samples studied by Barmby et al. (2002) for M 31 GCs and Holland et al. (1999) and Harris et al. (2002) for Cen A GCs, although it goes to somewhat larger values. The bump in the interval $e=0.35-0.4$ is not present in any of the samples described above, although $\mathrm{H} 2002$ find a cluster with $e=0.33$. Objects having this extreme elongation should 


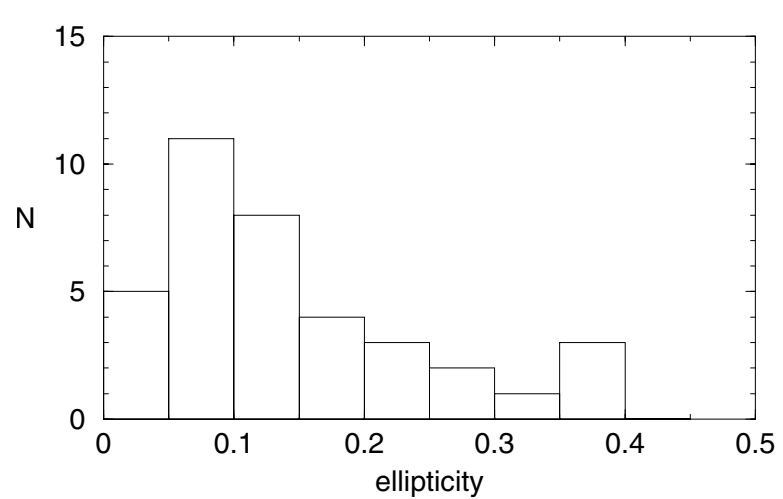

Fig. 5. Histogram of the ellipticities. The reader is referred to Fig. 7 of Harris et al. (2002) for a similar plot for other GCSs. The strikingly high ellipticities observed in a few cases in our sample put them among the most flattened GCs observed so far.

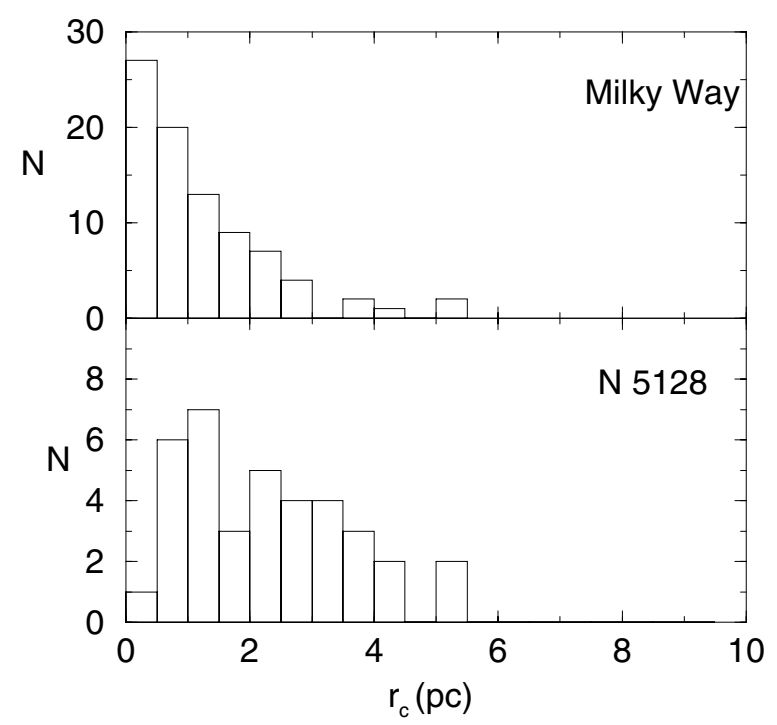

Fig. 6. Histogram of the core radius $r_{\mathrm{c}}$ for Galactic clusters (top panel, 85 objects) and NGC 5128 (bottom panel, 39 objects).

not necessarily be discarded as GCs. Indeed, the H2002 object is certainly a cluster. In addition, Larsen (2001), using HST images, studied a bright cluster in NGC 1023 and estimated its ellipticity as $0.37 \pm 0.01$, thus being one of the most flattened GCs known so far. On the other hand, we must recognize from the above discussion that galaxies certainly must contaminate our sample and these may well help to skew the distribution to higher ellipticity (see Sect. 5 for a discussion on the background contamination).

\subsection{Size, luminosity and surface brightness}

Both the effective radii $r_{\mathrm{e}}$ and core radii $r_{\mathrm{c}}$ are listed in Table 3. The core radii agree very well with the range of values for GCs in NGC 5128 given in Harris et al. (2002), and as can be seen from Fig. 6, are also well in the range of Galactic GCs. The dip observed at $r_{\mathrm{c}}<0.5 \mathrm{pc}$ is probably due to a selection effect and is also present in the $\mathrm{H} 2002$ sample, i.e. the known GCs tend to be the largest, most easily resolved.

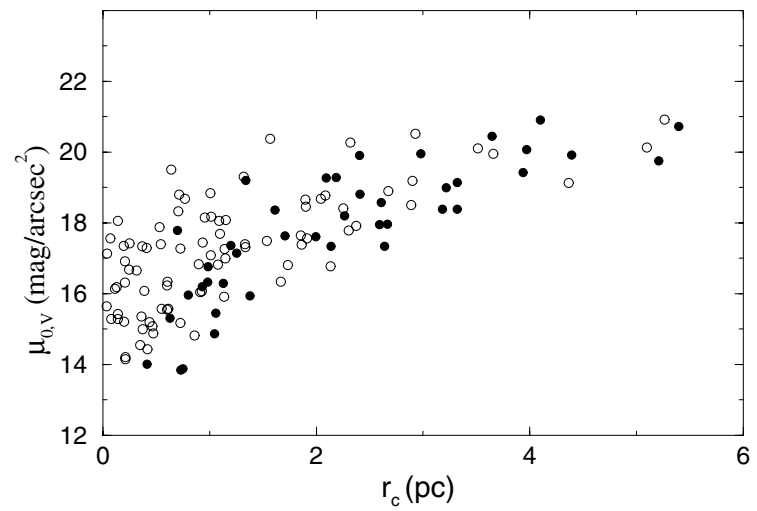

Fig. 7. Comparison of the central surface brightness in the $V$-band $\mu_{0}(V)$ vs. $r_{\mathrm{c}}$ for clusters in NGC 5128 (filled circles) and Galactic GCs (open circles).

Figure 7 compares the core radii and the central surface brightness $\mu_{0}(V)$ between clusters of NGC 5128 (filled circles) and Galactic ones (open circles). No major systematic difference is observed and both groups show a similar spread in their central surface brightness at a given $r_{\mathrm{c}}$. However, the tendency of the smaller clusters in NGC 5128 to have higher central surface brightness could indicate a selection bias. This is very plausible as our detection is based on the presence of residuals and faint compact clusters would be hardly noticeable.

Although the core radii are not surprising, the effective radii are generally somewhat larger than the typical 2-7 pc observed for MW GCs, with a few extreme clusters with $r_{\mathrm{e}} \sim 15 \mathrm{pc}$. However, clusters with sizes of $\sim 30$ pc have been observed in M 31 (Huxor et al. 2005) at galactocentric distances between 15 and $35 \mathrm{kpc}$. Again, we are certainly biassed against the smaller, more compact clusters.

In addition to these large GCs, Martini \& Ho (2004) have derived masses and sizes for a sample of 14 bright clusters in NGC 5128. Their masses are in the range $10^{6}-10^{7} M_{\odot}$ and thus some clusters are even more massive than some dwarf galaxies. In our Galaxy, the most massive GC ( $\omega$ Cen) may well be a stripped dwarf nucleus (see Martini \& Ho 2004, and references therein). A similar origin has been proposed for G1 in M 31 (e.g. Bekki \& Freeman 2003; Bekki \& Chiba 2004). By implication, these very massive NGC 5128 GCs may also have had such an origin. On the other hand, de Propris et al. (2005) have compared surface brightness profiles of the nuclei of Virgo dwarf galaxies with those of UCDs. They concluded that UCDs are more extended and brighter than the dwarf nuclei, so the "threshing scenario" is unlikely.

Whatever the physical processes that led to the formation of these very massive clusters, their location in $r_{\mathrm{h}}-M_{V}$ parameter space is quite different from what is observed for MW GCs, as is clear in Fig. 8. The solid line shows the equation derived by van den Bergh \& Mackey (2004) who found that only 2 MW GCs lie above this line: $\omega$ Cen and NGC 2419. The data have been primarily taken from Huxor et al. (2005), to which we have added the samples of de Propris et al. (2005), Richtler et al. (2005) and Haşegan et al. (2005). The references for each dataset and their symbols are given in Fig. 8. 


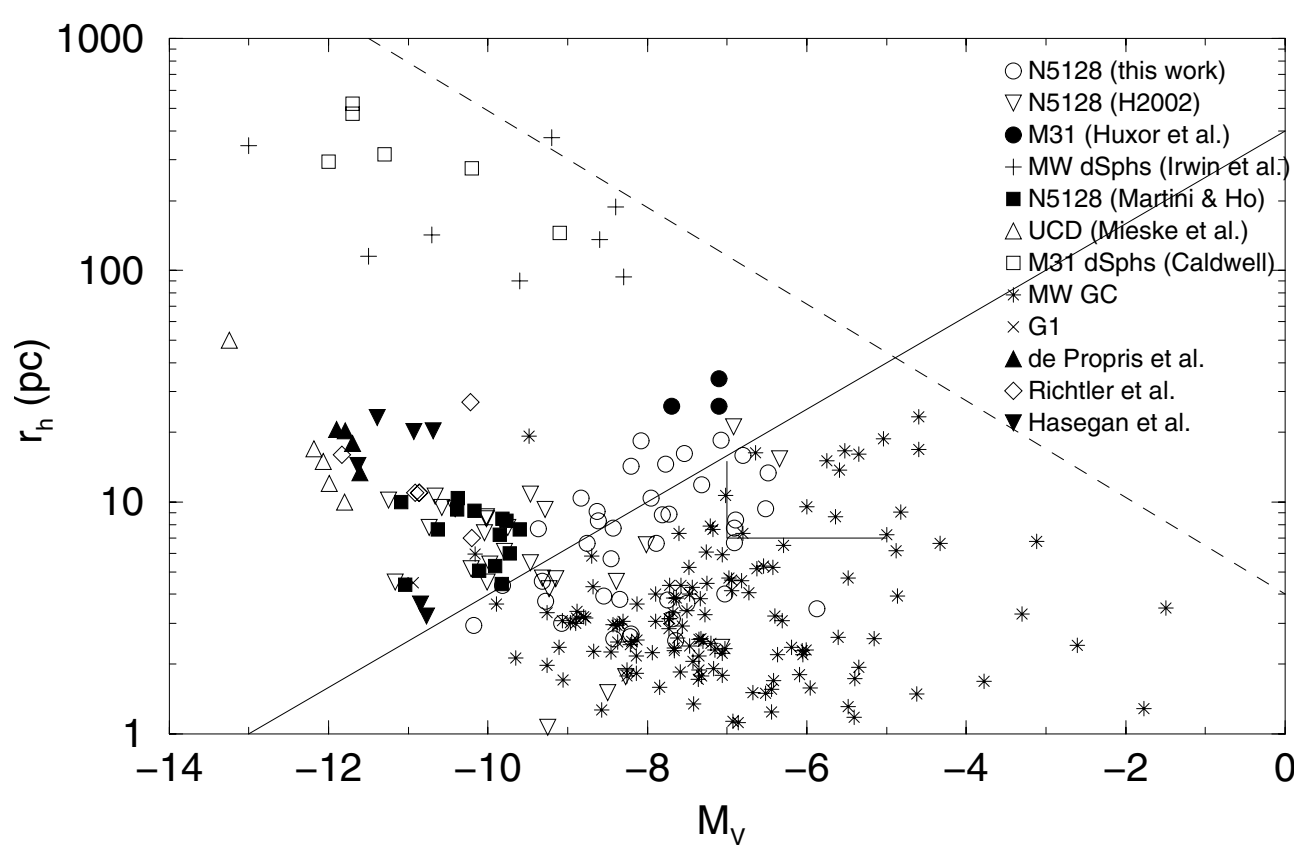

Fig. 8. Half-light radius $r_{\mathrm{h}}$ (in pc) versus $M_{V}$. A distance of $4 \mathrm{Mpc}$ for NGC 5128 has been assumed. The references for the different symbols follow. Open circles are the GCs analysed in this work. Filled circles are the luminous clusters found in the halo of M 31 by Huxor et al. (2005). Downward-pointing empty triangles represent the data for GCs in NGC 5128 using HST images from Harris et al. (2002). The plus signs are dwarf Spheroidals associated with the Milky Way (Irwin \& Hatzidimitriou 1995). The sample of massive clusters in NGC 5128 studied by Martini \& Ho (2004) are plotted using filled squares. Open squares show the dwarf Spheroidals associated with M 31 (Caldwell et al. 1992; and Caldwell 1999). UCDs from Mieske et al. (2002) are represented by the upward-pointing empty triangles. The MW GCs are the asterisks (data taken from the catalog of Harris 1996). G1 (=Mayall II), the brightest GC in M31, is shown with a cross. The data from de Propris et al. (2005) and Haşegan et al. (2005) are shown with the filled upward and downward pointing triangles, respectively. Finally, the empty diamonds represent the striking clusters/UCDs found by Richtler et al. (2005) around NGC 1399. The equation $\log r_{\mathrm{h}}=0.2 M_{V}+2.6$ from van den Bergh \& Mackey (2004) is the solid line and the dashed line shows a value of constant average surface brightness with $r_{\mathrm{h}}$. The solid L-shape indicates the region (above and to the right) where FFs are found. GCs form a continuum in this diagram and even approach the region occupied by UCDs.

In spite of the difference between the most massive NGC 5128 GCs and their MW counterparts, the most striking feature of this diagram is that there are essentially no longer any gaps in the distribution of the ensemble of GCs. When Huxor et al. first presented this diagram, they used it to point to the unique position of their newly-discovered M 31 clusters. However, the addition of our data and that of H2002 now nicely fills the gaps that were present in the Huxor et al. (2005) version. Many NGC 5128 GCs are found above the van den Bergh \& Mackey (2004) line. Clearly, this line no longer appears to have any special significance. In particular, we find a number of NGC 5128 clusters that are large and of intermediate luminosity, falling only slightly below the Huxor et al. M31 clusters. In addition, several NGC 5128 clusters are found in the region formerly inhabited almost exclusively by the Faint Fuzzies of Larsen \& Brodie (2000). Note that the majority of the NGC 5128 clusters in this region are also red and presumably metal-rich, as are the Faint Fuzzies. The 3 M 31 clusters, on the other hand, are rather blue. Therefore, large, faint clusters are not exclusive to lenticular galaxies. However, note that FFs are also distinguished by their disk-like kinematics. This cannot be further studied in our sample without spectroscopic observations and we are in the process of obtaining such data.

Thus, our data serve to illustrate that, although they exhibit a range of 1-2 orders of magnitude in both luminosity and size,
GCs can not be broken down into well-separated, distinct subtypes but instead form a continuum. This is in keeping with the assessment of Larsen (2002). This continuum now extends to the realm of the Ultra Compact Dwarfs (e.g. Hilker et al. 1999; Drinkwater et al. 2002), lending support to the idea that these objects are indeed similar (Martini \& Ho 2004) and have similar origins (Mieske et al. 2002). Note that Haşegan et al. (2005) find several objects in their Virgo Cluster ACS survey that they term "dwarf-globular transition objects" (DGTOs). Several of these fall close to the main locus of GCs in Fig. 8 and several lie closer to that of previously identified UCDs. The existence of such objects further serves to fill in the parameter space in this diagram. Note, however, that Haşegan et al. (2005) suggest that at least UCDs and GCs may be best distinguished via other parameters, e.g. $\mathrm{M} / \mathrm{L}$ ratio.

\subsection{Correlations with galactocentric distance}

Several of the structural parameters may depend on the galactocentric distance $R_{\mathrm{gc}}$, including the size, brightness and concentration. For the Milky Way system, van den Bergh et al. (1991) have noticed that $r_{\mathrm{h}}$ is correlated with $R_{\mathrm{gc}}$, with larger clusters observed at larger distances.

A similar correlation was observed for the GCS of M31 (Barmby et al. 2002), but it is not so clear for the H2002 


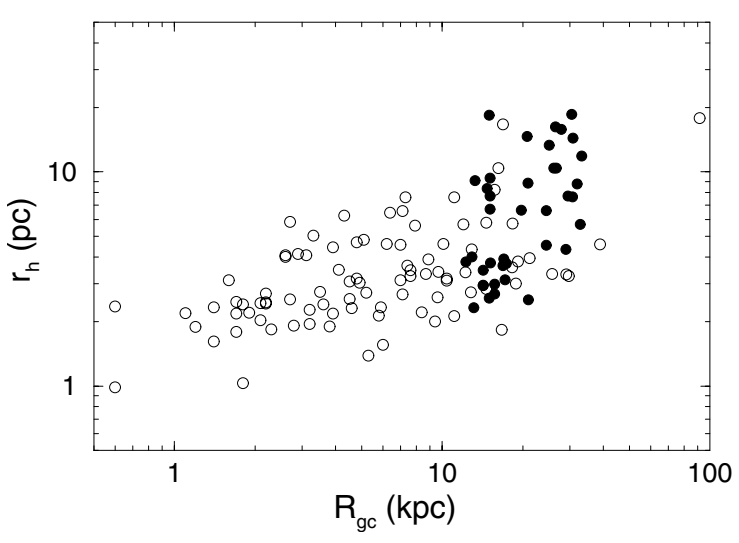

Fig. 9. Dependence of the half-light radius $r_{\mathrm{h}}$ on the projected distance of the clusters to the optical center of NGC 5128 (filled circles). A distance of $4 \mathrm{Mpc}$ has been assumed for the galaxy. For comparison, the open circles represent Milky Way GC, taken from the catalog of Harris (1996).

sample. This is in part, because of the different cameras used for inner and outer clusters and, most important, because of projection effects over a small sample, which can blur any subtle trend with the actual, three-dimensional galactocentric distance.

Our data show, nevertheless, a clear agreement with the trend defined by the Galactic GCs (see Fig. 9). On the logarithmic scale, it is apparent that the observed range of $r_{\mathrm{h}}$ in NGC 5128 matches that in the Milky Way, although our clusters have on average larger projected distances (see also Fig. 1). More clusters at smaller distances need to be observed before a better comparison can be made. Regarding the luminosity, we do not see any correlation with $R_{\mathrm{gc}}$. In analogy to Fig. 10 of $\mathrm{H} 2002$, we searched for possible correlations between the concentration parameter and ellipticity with $R_{\mathrm{gc}}$, but none were observed.

\subsection{Metallicity and size}

To estimate the metallicity of the clusters, we have used the relation from Harris \& Harris (2002) between the Washington system $\left(C-T_{1}\right)$ colour and metallicity, and assumed a uniform reddening of $E(B-V)=0.11$ (Schlegel et al. 1998) for all clusters. Given the very large distances of our clusters from the central dust lane, this assumption should be valid. No trend is seen between the derived metallicities and $R_{\mathrm{gc}}$, but due to the still small sample, we do not further comment on this. Instead, we have arbitrarily divided the sample into metal-poor and metal-rich at $\mathrm{Fe} / \mathrm{H}=-1$ (see Harris et al. 2004a) and looked for systematic differences in the cluster size. The analysis performed on a number of galaxies (see Kundu et al. 1999; Kundu \& Whitmore 2001; Larsen et al. 2001; Jordán 2004; Jordán et al. 2005) indicate that metal-poor (blue) clusters have mean half-light radii $\sim 20 \%$ larger than those of metal-rich (red) ones. However, this is not the case for the $\mathrm{H} 2002$ sample. Using the same metallicity cut, they find mean radii of $r_{\mathrm{h}}=7.37 \pm 1.03$ and $r_{\mathrm{h}}=7.14 \pm 0.76$ for the metal-poor and metal-rich groups respectively, i.e. indistinguishable.

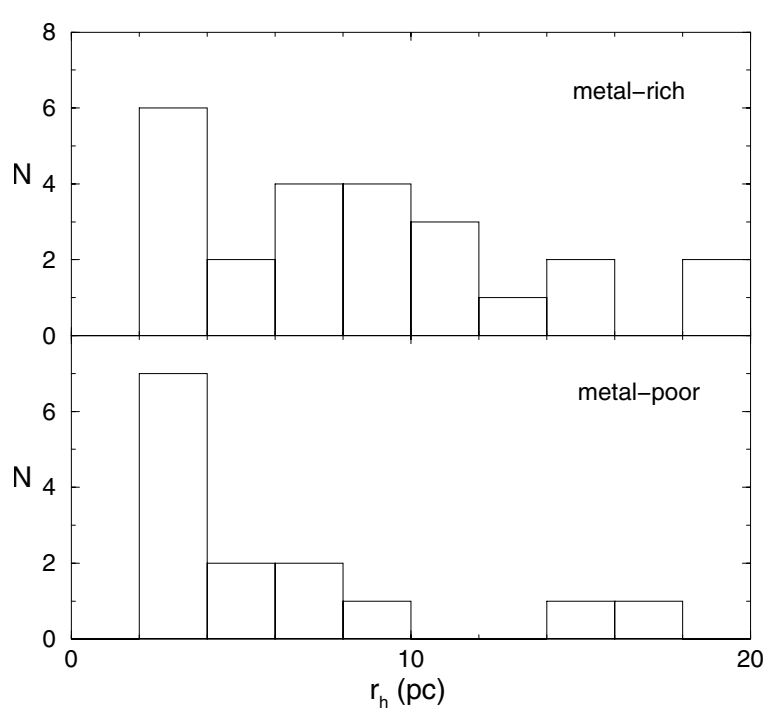

Fig. 10. Half-light radii $r_{\mathrm{h}}$ for the metal-poor and metal-rich subgroups. The metallicity is estimated from the Washington $C-T 1$ colour according to Harris \& Harris (2002) and the subgroups are divided at $[\mathrm{Fe} / \mathrm{H}]=-1$.

Our results are in better agreement with those of H2002 than with other studies. Figure 10 shows the histogram of halflight radii $r_{\mathrm{h}}$ for both subpopulations and it is clear that, if a relation between metallicity and cluster size exists, then it is opposite to that reported by Jordán (2004) and most previous WFPC2 studies of barely resolved clusters. The returned median values are $r_{\mathrm{h}}=4.1 \pm 1.2$ and $r_{\mathrm{h}}=8.0 \pm 0.9 \mathrm{pc}$ for the metal-poor and metal-rich subgroups respectively. The paucity of large metal-poor clusters is apparent. It is striking that the Barmby et al. (2002) histogram of $r_{\mathrm{h}}$ for M $31 \mathrm{GCs}$ is broader for the metal-poor than for the metal-rich sample, which is contrary to what we see. This holds also if metallicities via broadband colours are included.

The general agreement of our results with those of H2002 lends credence to our findings. Although the combined sample size is small, these data are the best available in terms of number of pixels per resolved image and the results should be the most robust. The possibility exists that some background galaxies still contaminate our sample and only a few of them would be needed to affect the shape of the size histograms. However, galaxy contamination should only be a small effect (see Sect. 5). In fact, contaminating galaxies from the Woodley et al. (2005) sample are preferentially in the blue (metal-poor) part of our GC colour selection. This is opposite to the effect required to explain the size difference between metal-rich and metal-poor clusters by galaxy contamination. Another possibility is that clusters in NGC 5128 are different from those observed in other galaxies. Clearly, more observations are required to resolve this intriguing issue.

\subsection{Total cluster population}

Harris et al. (2004b) found that the NGC 5128 GCs follow a steep projected radial distribution, of the form $\sigma \sim r^{-2}$, and that the clusters were mostly confined to the inner $\sim 10^{\prime}$. However, 
note that all of our fields are beyond this radius, extending from $11^{\prime}$ out to $29^{\prime}$, with most outside $15^{\prime}$. In the 30 fields we observed, we confirmed the GC nature of $1 / 3$ of the 44 original Harris et al. (2004a) candidates and found 22 other GC candidates, roughly 1 per field. The success of our GC search in these distant regions of the galaxy's halo leads us to believe both that the radial extent of the NGC 5128 GC population is indeed large, with a significant population out to $30^{\prime}$ and beyond, and that the number of clusters derived by Harris et al. (2004a) may be an underestimate. A crude calculation derives a mean GC surface density for our sample that agrees well with the value derived by Harris et al. (2004b), at the mean galactocentric distance of our sample. However, our sample only includes a few of the known GCs at this radius. In addition, for their total population calculation, Harris et al. (2004b) assumed a value derived from the halo light profile, which fits well their GC profile out to $\sim 13^{\prime}$ but beyond this their GC densities appear higher than those of the galaxy itself, as is generally observed. If their surface density of clusters beyond $\sim 10^{\prime}$ is only slightly underestimated, this could lead to a significant increase in their total GC population estimate of $\sim 1000$ clusters and this indeed appears to be the case.

Clearly, the final census of NGC 5128 GCs will not be achieved until a complete search of the outer halo to at least $45^{\prime}$ is conducted. Radial velocity surveys would be very inefficient and time-consuming given the huge $(\sim 100$ to 1$)$ ratio of field interlopers to genuine GCs. For optimum efficiency, such a search requires both the widest field coverage combined with very high spatial resolution in order to resolve the clusters. Such an optimum combination is provided by the IMACS instrument at Magellan and we are in the process of obtaining these data.

\section{Background contamination}

Certainly, even after the selection criteria applied to our cluster candidates, a (hopefully small) fraction of our objects consists of background galaxies. Foreground stars can be surely discarded as part of the contaminating population, because they cannot be observed as extended objects in our frames. The purpose of this section is to give an idea of the contamination level and its effects on our results.

\subsection{Effects of including galaxies in our sample}

To see how galaxies behave in our diagrams (i.e. Figs. 7 and 8), we have re-analysed our frames and selected objects which are either spectroscopically confirmed background galaxies or fall without doubt into this category after visual inspection. All of the latter are well resolved objects, at least twice as large as the clusters and many had substructures like disks or spiral arms. Fainter objects were included only if they were spectroscopically confirmed galaxies. This is the case for WHH094, WHH127 and WHH43, all from Woodley et al. (2005).

Our images of these galaxies were analysed in exactly the same way as with the cluster candidates, i.e. light profiles were fitted via ishape and using the same stellar PSF for the corresponding frame. The fitting radius and all parameters were
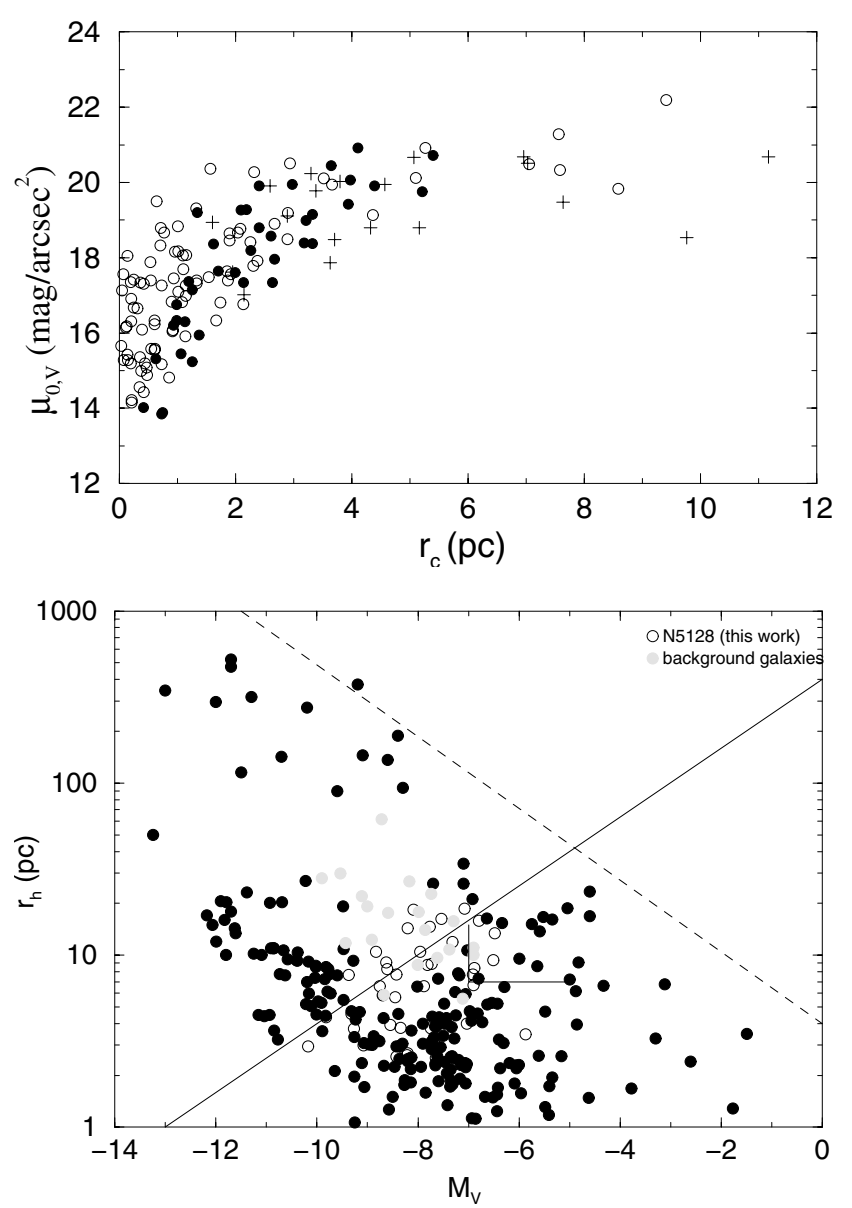

Fig. 11. Upper panel: same as Fig. 7 with background galaxies represented by the plus sign. Besides the tendency to have larger $r_{\mathrm{c}}$ and perhaps lower surface brightnesses than the NGC 5128 (filled circles) and MW (open circles) GCs, galaxies cannot be clearly distinguished in this space. Lower panel: same as Fig. 8, but the analysed sample of galaxies has been included and is represented by the grey circles. Open circles are the GC candidates and filled circles represent all objects from the references given in Fig. 8.

handled as was done for the cluster candidates, the only difference is that we are now working with a sample of purely galaxies, so "normal" Washington colours and magnitudes and reasonable fits with King profiles (i.e. little residual in the subtracted image) are not expected.

The derived structural parameters were then combined with the photometry from Harris et al. (2004a). After correcting for aperture size, reddening and forcing the galaxies to be at the assumed distance for NGC 5128 (4 Mpc), we derived their surface brightnesses (a quantity which is anyway independent of the distance), absolute magnitudes, half-light and core radii in parsecs, etc.

Strikingly, the King profile did in fact fit quite well to the majority of galaxies for which no substructure was visible, at least within the inner 25 pixels. For disky or spiral galaxies, the residuals were clear. Moreover, the galaxies occupy a similar region as the GCs in the $\mu_{0}-r_{\mathrm{c}}$ parameter space, as Fig. 11 shows (upper panel), with perhaps a reasonable tendency to larger sizes and lower surface brightnesses. We stress that, in many aspects, the comparison between galaxies and clusters 
based on their structural parameters has no physical meaning. In this case, the derivation of $r_{\mathrm{c}}$ via a King profile is not more than an artifact but it is adequate for the purpose of assessing the contamination level and to illustrate its behaviour.

Similarly, there is little observed difference in the $r_{\mathrm{h}}-M_{V}$ space (Fig. 11, lower panel). Indeed, the galaxies extend to higher sizes and span a region which is a bit above "normal" GCs in NGC 5128 and in our Galaxy in this logarithmic scale. Note, however, that the smallest galaxies in our sample (again, small in the sense of $r_{\mathrm{h}}$ obtained by brute force) is comparable to the mid-size/large clusters. Truly large galaxies have no counterparts in our sample of GCs.

\subsection{The level of contamination}

To assess the level at which our diagrams and results could be affected by contamination is the aim of this section.

Without doubt, the most reliable way to determine if a cluster candidate is actually a background galaxy is via spectroscopy. Nevertheless, an independent image-based analysis can also be used as a reasonable first measurement. For this, we made use of images taken independently with the Gemini $8 \mathrm{~m}$ Telescope and the GMOS Camera (Harris et al. 2006). These are of poorer seeing than the MagIC frames $\left(0.7^{\prime \prime}-0.8^{\prime \prime}\right)$ and cover the central region of NGC 5128, so there are no common objects. However, the published spectroscopic work concentrates almost solely in this region and therefore, dozens of confirmed clusters and galaxies are available for study.

We apply the same reduction, analysis and selection criteria to the cluster-like candidates within these GMOS fields and look for existing velocities for this sample. Of course, this is only meaningful if one does not know a priori which objects are the clusters. Thus, the correlation with spectra was done as the very last step.

First, the stellar PSF was made from 20-30 stars per frame. Then, this PSF was subtracted from all objects and GC candidates were chosen among those which left a "doughnut" shaped residual, exactly as was done for our original sample. After this, we looked for their Washington photometry in H2004 and applied the same colour and magnitude cuts. We then ran ishape again with the same parameters as before and in exactly the same way.

All in all, we obtained 87 cluster-like objects for which structural parameters could be derived and which passed the selection criteria. 42 of these had been previously observed spectroscopically (Peng et al. 2004; and Woodley et al. 2005). 39 of the 42 GC candidates turned out to be clusters and 3 are actually background galaxies. There are, in addition, several GCs which we failed to detect, presumably due to the low resolution of the images and their compactness.

This shows that the galaxy contamination level is roughly $7 \%$ for the GMOS images and can be even lower for the MagIC study, which benefitted from much better resolution.

On the one hand, we have shown that background galaxies cannot be easily told from the clusters based on their structural parameters and location in the diagrams, so that the risk exists if no careful selection is performed, although in this case one still should not be led to erroneous conclusions since the properties are similar. On the other hand, an independent test shows that this contamination is at most $7 \%$ when the morphology and Washington colours are used as selection criteria. Thus, our results reflect by far the behaviour of GCs and not background galaxies. Note that the ratio of galaxies to GCs certainly increases with galactocentric distance, due to the strong central concentration of the latter, and thus one might naively expect that the contamination level of our sample may be higher since it is more distant than the GMOS fields. However, we have shown that, given sufficiently good seeing and our analysis techniques, one should be able to maintain the contamination to a low level irrespective of galactocentric distance.

As explained in Sect. 2.3, Woodley et al. (2005) find that two of our cluster candidates are actually background galaxies. For one of them (HHG94), our much better seeing shows that it is actually a cluster candidate projected $1.6^{\prime \prime}$ from a galaxy observed. This separation is close to the typical seeing for the Harris et al. (2004a) database, which was the basis of the Woodley et al. (2005) work. It is therefore reasonable that the velocity obtained corresponds actually to that of the galaxy, or a composite of the two velocities.

If this is indeed the case, then there is only one galaxy in the common sample of 6 cluster candidates, which is roughly what we should expect from our results, given the small numbers involved. Our GMOS test is more definitive, so we give more weight to its result and estimate our final galaxy contamination level at about $10 \%$.

\section{Conclusions}

We have obtained very high spatial resolution images in excellent seeing conditions with the Magellan telescope + MagIC camera of 44 GC candidates in the outer regions of NGC 5128 from the list provided by Harris et al. (2004a). These data not only allow us to determine the nature of these candidates via spatial resolution but also allow us to derive their detailed structural parameters. This is the first time such parameters are determined for GCs beyond the Local Group from groundbased images. About one third of the candidates appear to be bonafide GCs. In addition, we serendipitously discovered 18 new GC candidates and also derived their structural parameters.

We compare our cluster sample in detail with those of other GC samples in other galaxies with similar information available. We find that, in general, our clusters are similar in size, ellipticity, core radius and central surface brightness to their counterparts in other galaxies, in particular those in NGC 5128 observed with HST by Harris et al. (2002). However, our clusters extend to higher ellipticities and larger half-light radii than their Galactic counterparts, confirming the results of H2002. Combining our results with those of Harris et al. (2002) fills in the gaps previously existing in the $r_{\mathrm{h}}-M_{V}$ parameter space and indicates that any substantial difference between presumed distinct cluster types in this diagram, including for example the Faint Fuzzies of Larsen \& Brodie (2000) and the "extended, luminous" M31 clusters of Huxor et al. (2005) is now removed and that clusters form a continuum. Indeed, this continuum now extends to the realm of the Ultra Compact Dwarfs. 
The metal-rich clusters in our sample have half-light radii in the mean that are almost twice as large as their metal-poor counterparts, at odds with the generally accepted trend. Finally, our discovery of a substantial number of new cluster candidates in the relatively distant regions of the NGC 5128 halo suggest that current values of the total number of globular clusters may be underestimates.

We have performed extensive tests to study the effect of background galaxies on our results and the expected amount of contamination. They show that galaxies and clusters cannot be clearly distinguished from their loci in the $r_{\mathrm{h}}-M_{V}$ and $\mu_{0, V}-$ $r_{\mathrm{c}}$ parameter spaces. However, if high resolution images are combined with an appropiate colour index like the Washington $\left(C-T_{1}\right)$, the level at which the GC sample is contaminated by background galaxies is about $10 \%$. Therefore, we expect that our results reflect largely the physical properties of actual clusters rather than background galaxies.

Acknowledgements. M.G. thanks Søren Larsen for his help with ishape and comments, as well as Avon Huxor for the data in Fig. 8. D.G. gratefully acknowledges support from the Chilean Centro de Astrofísica FONDAP No. 15010003. We thank the referee for his/her comments and suggestions which greatly improved this paper, especially the discussion about the galaxy contamination.

\section{References}

Aarseth, S. J., \& Heggie, D. C. 1998, MNRAS, 297, 794

Barmby, P., Holland, S., \& Huchra, J. P. 2002, AJ, 123, 1937

Bekki, K., \& Freeman, K. C. 2003, MNRAS, 346, L11

Bekki, K., \& Chiba, M. 2004, A\&A, 417, 437

Burkert, A., Brodie, J., \& Larsen, S. S. 2005, ApJ, 628, 231

Caldwell, N., Armandroff, T. E., Seitzer, P., \& Da Costa, G. S. 1992, AJ, 103, 840

Caldwell, N. 1999, AJ, 118, 1230

de Propris, R., Phillipps, S., Drinkwater, M. J., et al. 2005, ApJ, 623, L105

Dirsch, B., Richtler, T., Geisler, D., et al. 2003, AJ, 125, 1908

Structure and Dynamics of Globular Clusters. Proceedings of a Workshop held in Berkeley, California, July 15-17, 1992, ed. S. G. Djorgovski, \& G. Meylan (California: San Francisco, ASP), 1993, 50

Djorgovski, S. 1995, ApJ, 438, 29L

Djorgovski, S., Gal, R., McCarthy, J., et al. 1997, ApJ, 474, L19

Drinkwater, M., Bekki, K., Couch, W., et al. 2002, IAU Symp., 207, 287

Elson, Fall, \& Freeman 1987, ApJ, 323, 54

Fleming, D. E. B., Harris, W. E., Pritchet, C. J., \& Hanes, D. A. 1995 , AJ, 109, 1044

Frenk, C. S., \& Fall, S. M. 1982, MNRAS, 199, 565

Geisler, D., \& Hodge, P. 1980, ApJ, 242, 66

Geisler, D. 1996, AJ, 111, 480

Geisler, D., Lee, M. G., \& Kim, E. 1996, AJ, 111, 1529

Graham, J. A., \& Phillips, M. M. 1980, ApJ, 239, L97
Haşegan, M., Jordán, A., Côté, P., et al. 2005, ApJ, 627, 203

Harris, G. L. H., Geisler, D., Harris, H. C., \& Hesser, J. E. 1992, AJ, 104,613

Harris, W. E. 1996, AJ, 112, 1487

Harris, W. E., \& Harris, G. L. H. 2002, AJ, 123, 3108

Harris, W. E., Harris, G. L. H., Holland, S., \& McLaughlin, D. 2002, AJ, 124, 1435

Harris, G., Geisler, D., \& Harris, W. E. 2004a, AJ, 128, 712

Harris, G., Harris, W. E., Geisler, D., et al. 2004b, AJ, 128, 723

Hesser, J. E., Harris, H. C., van den Bergh, S., \& Harris, G. L. H. 1984, ApJ, 276, 491

Hilker, M., Infante, L., Vieira, G., Kissler-Patig, M., \& Richtler, T. 1999, A\&AS, 134, 75

Holland, S., Côté, P., \& Hesser, J. E. 1999, A\&A, 348, 418

Huxor, A., Tanvir, N. R., Irwin, M. J., et al. 2005, MNRAS, 360, 1007

Irwin, M., \& Hatzidimitriou, D. 1995, MNRAS, 277, 1354

Israel, F. P. 1998, A\&ARv, 8, 237

Jordán, A. 2004, ApJ, 613, L117

Jordán, A., Côté, P., Blakeslee, J. P., et al. 2005, ApJ, in press [arXiv: astro-ph/0508219]

King, I. 1962, AJ, 67, 471

Kissler-Patig, M. 2000, RvMA, 13, 13

Kundu, A., Whitmore, B. C., Sparks, W. B., et al. 1999, ApJ, 513, 733

Kundu, A., \& Whitmore, B. C. 2001, AJ, 121, 2950

Larsen, S. S. 1999, A\&AS, 139, 393

Larsen, S. S., \& Brodie, J. P. 2000, AJ, 120, 2938

Larsen, S. S. 2001, AJ, 122, 1782

Larsen, S. S., Brodie, J. P., Huchra, J. P., Forbes, D. A., \& Grillmair, C. J. 2001, AJ, 121, 2974

Larsen, S. S., Brodie, J. P., Sarajedini, A., \& Huchra, J. P. 2002 AJ, 124,2615

Larsen, S. S. 2002, IAU Symp., 207

Larsen, S. S., \& Brodie, J. P. 2003, ApJ, 593, 340

Larsen, S. S. 2004, An ISHAPE user's guide, http://www . astro.ku.dk/ soeren/baolab

Layden, A. C., \& Sarajedini, A. 2000, AJ, 119, 1760

Mackey, A. D., \& Gilmore, G. F. 2003, MNRAS, 340, 175

Mackey, A. D., \& Gilmore, G. F. 2004, MNRAS, 355, 504

Martini, P., \& Ho, L. C. 2004, ApJ, 610, 233

Mieske, S., Hilker, M., \& Infante, L. 2002, A\&A, 383, 823

Peng, E. W., Ford, H. C., \& Freeman, K. C. 2004, ApJS, 150, 367

Phillips, M. M. 2002, http://www.lco.cl/lco/magellan/instruments/ MAGIC/iraf_reductions.html

Rejkuba, M. 2004, A\&A, 413, 903

Rich, R. M., Mighell, K. J., Freedman, W. L., \& Neill, J. D. 1996, AJ, 111,768

Richtler, T., Dirsch, B., Larsen, S., Hilker, M., \& Infante, L. 2005, A\&A, 439, 533

Schlegel, D. J., Finkbeiner, D. P., \& Davis, M. 1998, ApJ, 500, 525

Soria, R., Mould, J. R., Watson, A. M., et al. 1996, ApJ, 465, 79

Spitzer, L. Jr., \& Thuan, T. X. 1972, ApJ, 175, 31

Stetson, P. B. 1987, PASP, 99, 191

van den Bergh, S. 1984, PASP, 96, 329

van den Bergh, S., Morbey, C., \& Padzer, J. 1991, ApJ, 375, 594

van den Bergh, S., \& Mackey, A. D. 2004, MNRAS, 354, 713

Woodley, K. A., Harris, W. E., \& Harris, G. L. H. 2005, AJ, 129, 2654 